\title{
Article \\ Symptom Profiles, Health-Related Quality of Life, and Clinical Blood Markers among Korean Community-Dwelling Older Adults Living with Chronic Conditions
}

\author{
Jongmin Park ${ }^{1}\left(\mathbb{D}\right.$, Nada Lukkahatai ${ }^{2}\left(\mathbb{D}\right.$, Nancy Perrin $^{2}$, Yoonju Kim ${ }^{3}$, Leorey N. Saligan ${ }^{4}(\mathbb{D}$ \\ and Chang Won Won ${ }^{5, *(1)}$ \\ College of Nursing, Pusan National University, Gyeongsangnam-do 50612, Korea; jmpark@pusan.ac.kr \\ 2 School of Nursing, Johns Hopkins University, Baltimore, MD 21218, USA; nada.lukkahatai@jhu.edu (N.L.); \\ nperrin@jhu.edu (N.P.) \\ 3 Department of Nursing, Graduate School, Kyung Hee University, Seoul 02447, Korea; \\ kimyoonju29@gmail.com \\ 4 National Institute of Nursing Research, National Institutes of Health, Bethesda, MD 20892, USA; \\ saliganl@mail.nih.gov \\ 5 Elderly Frailty Research Center, Department of Family Medicine, College of Medicine, Kyung Hee University, \\ Seoul 02447, Korea \\ * Correspondence: chunwon62@naver.com; Tel.: +82-2-958-8700; Fax: +82-2-958-8699
}

check for updates

Citation: Park, J.; Lukkahatai, N.; Perrin, N.; Kim, Y.; Saligan, L.N.; Won, C.W. Symptom Profiles, Health-Related Quality of Life, and Clinical Blood Markers among Korean Community-Dwelling Older Adults Living with Chronic Conditions. Int. J. Environ. Res. Public Health 2021, 18, 1745. https:// doi.org/10.3390/ijerph18041745

Academic Editor: Paul B. Tchounwou

Received: 7 January 2021

Accepted: 9 February 2021

Published: 11 February 2021

Publisher's Note: MDPI stays neutral with regard to jurisdictional claims in published maps and institutional affiliations.

Copyright: (c) 2021 by the authors. Licensee MDPI, Basel, Switzerland. This article is an open access article distributed under the terms and conditions of the Creative Commons Attribution (CC BY) license (https:// creativecommons.org/licenses/by/ $4.0 /)$.
Abstract: Older adults suffer from multiple symptoms, which negatively affects their health-related quality of life. The single-symptom management approach has been less than effective. The data of 2362 Korean community-dwelling older adults aged 70 and above were analyzed in the Korean Frailty and Aging Cohort Study (KFACS) study. A cluster analysis, correlation analysis, and logistic regression were used to analyze the data. We found three symptom clusters: high symptom burden (HSB, $n=1032$ ); pain and fatigue group (PAF, $n=566$ ); and the sleep deprivation group (SDP, $n=764$ ). Participants in the HSB group are more likely to be of old age $(\mathrm{OR}=1.1)$, be female $(\mathrm{OR}=2.4)$, live in a rural area $(\mathrm{OR}=1.4)$, have low physical activity $(\mathrm{OR}=0.9)$, and have multiple chronic conditions $(\mathrm{OR}=1.5)$. The clinical blood markers analysis showed a negative relationship among the physical health, free T4 $(r=-0.083, p<0.01)$ and insulin $(r=-0.084, p<0.01)$. The sex-specific blood markers analysis showed differences among three clusters. While free testosterone (male: $r=0.124$, female: $r=0.110, p<0.05$ ) and dehydroepiandrosterone (DHEA) (male: $r=0.352$ and female: $r=0.134, p<0.05$ ) were associated with physical health in the HSB group, only free testosterone was associated with mental health (male: $r=-0.093$, and female: $r=-0.116, p<0.05$ ) in the SDP group. These findings suggest the potential role of the patient's sex and sex hormones in symptoms of Korean community-dwelling older adults. Understanding the symptom profiles and impact of biopsychosocial factors may enhance precision symptom management.

Keywords: symptom cluster; quality of life; biomarkers; chronic conditions; community-dwelling older adults

\section{Introduction}

In 2019, the total aging population in the world was 703 million, which is expected to double by 2050 [1]. As life expectancy increases and the birth rate decreases, the aging population is growing in South Korea. By 2065, the proportion of the elderly will increase three-fold, from $13 \%$ in 2015 [2]. Nearly $80 \%$ of Korean older adults have at least one chronic condition, including cardiovascular disease, musculoskeletal disease, or diabetes mellitus. With chronic conditions and the aging process, older adults suffer from multiple symptoms such as pain, fatigue, sleep deprivation, and depression [3-5]. The intensity of these symptoms negatively affects older adults' health-related quality of life (HRQoL) [6,7]. 
Studies suggest that older adults with severe symptom burdens and poor physical function are at risk of hospitalization and nursing home admission [8-12].

The typical approach for symptom management among older adults has been to suggest an intervention based on each individual symptom. This "single symptom" approach results in complicated self-management as older adults and families are required to balance multiple medications, behavioral changes, cognitive coping strategies or physical interventions to manage all symptoms [13]. Many intervention studies (e.g., psychological interventions, physical activity programs, educational programs, massage, acupressure/acupuncture interventions) often find the effect of the interventions on more than one symptom. A symptom cluster is defined as the co-occurrence of two or more symptoms, which may or may not share the same underlying etiology [14]. The identification of symptom clusters in older adults with chronic conditions can be used to develop a simple, personalized, symptom cluster management program [12].

Recent studies suggest the role of inflammatory and endocrine clinical blood markers, including inflammatory cytokines, C-reactive protein, and dehydroepiandrosterone (DHEA) in the development and chronicity of symptoms, particularly with mobility limitation, depression, sleep deprivation, fatigue, and HRQoL [15-21]. To the best of our knowledge, there is limited evidence on the associations of these clinical blood markers with symptom burden and HRQoL. Investigating the role of these clinical blood markers will increase our understanding of the role of these markers on symptom burden and HRQoL among older adults.

This study aimed to (1) identify symptom clusters based on pain, mobility limitation, fatigue, and sleep deprivation among community-dwelling Korean older adults living with chronic conditions, and (2) investigate the relationships among symptom cluster profiles with HRQoL and clinical blood markers.

\section{Materials and Methods}

\subsection{Study Population}

This is an analysis of baseline data collected from the Korean Frailty and Aging Cohort Study (KFACS), which recruited 3014 community-dwelling older adults aged 70 and older from 10 nationwide hospitals and community health centers in South Korea from 2016 to 2017 [22]. Participants were asked to complete self-reported questionnaires related to socioeconomic status, living environment, health history, health-related behaviors, comorbidities, symptom experiences, and HRQoL. Laboratory tests were conducted to obtain clinical blood marker results. The present study's inclusion criteria were: (a) individual age 70 years and older, and (b) diagnosed with at least one chronic condition (i.e., cardiovascular disease, cerebrovascular disease, musculoskeletal disease, respiratory disease, gastrointestinal disease, renal disease, endocrine disease, or cancer). Participants who had difficulty communicating, visiting the healthcare center, a history of acute stroke or myocardial infarction during the past six months, or systolic blood pressure over $180 \mathrm{mmHg}$ were excluded from this study.

\subsection{Measures}

\subsubsection{Demographic Characteristics}

Demographic data, including age, sex, smoking history, alcohol consumption, education, marital status, and living status were obtained from the self-reported questionnaire. Body mass index (BMI) was calculated $\left(\mathrm{kg} / \mathrm{m}^{2}\right)$; overweight was defined as a BMI of $\geq 23 \mathrm{~kg} / \mathrm{m}^{2}$ [23]. Self-reported physical activities (vigorous, moderate, and light) within the past seven days for at least 10 minutes at a time were measured.

\subsubsection{Symptom Experience}

Pain was assessed by a single item: "How much pain do you have today?" Responses were measured as zero (0) "absence of pain" or one (1) "presence of pain." 
Mobility limitation was measured by the physical functioning (PF) scale, which consisted of 5 items on mobility and self-care [24]. The total scores ranged from 0 to 100, and higher ratings indicated better physical function. The subscale had good internal consistency with Cronbach's alpha $=0.89$ [24] and 0.82. The mobility score of 75.8 [24], was used as a cutoff score for determining participants with "no mobility limitation (0)" and "have mobility limitation (1)".

Fatigue was measured by a single item: "Have you ever felt tired in the past month?" The response was measured as zero (0) "absence of fatigue" or one (1) "presence of fatigue."

Sleep was measured by a single question: "How many minutes of actual sleep do you get at night?" The cutoff sleeping time of 6 hours/day was used to recode this variable into "no sleep deprivation (0)" if a participant slept $6 \mathrm{~h}$ or more, and "experienced sleep deprivation (1)" if a participant slept less than $6 \mathrm{~h}$ [25].

\subsubsection{Health-Related Quality of Life}

HRQoL was measured by the 12-Item Short-Form Health Survey (SF-12), which was developed to be a shorter version of SF-36, which is the most commonly used as a generic health status questionnaire [26]. The questionnaire consists of 12 items with eight concepts: physical functioning (two items), role-physical (two items), bodily pain (one item), general health (one item), vitality (one item), social functioning (one item), role-emotional (two items), and mental health (two items). The summary scales are referred to as the physical component summary (PCS-12) and the mental component summary (MCS-12) scale scores. Higher scores indicate better HRQoL. Cronbach's alpha for PCS-12 and MCS-12 in the general Korean population was 0.83 and 0.79 [27] and in our study was 0.84 and 0.82 , respectively.

\subsubsection{Clinical Blood Markers}

Participants' peripheral blood samples were taken at about 08:00 a.m. after $8 \mathrm{~h}$ of fasting. All blood samples at the ten centers were taken to a commercial laboratory for analysis. The white blood cell (WBC) count was measured using Cellpack DCL diluent (Sysmex, Co., Kobe, Japan) on an XN-9000 hematology analyzer (Sysmex Co., Kobe, Japan). The concentration of high-sensitivity C-reactive protein (hs-CRP) was determined using CRPHS reagent (Roche, Mannheim, Germany) on a Cobas 8000 C702 (Roche, Mannheim, Germany). Thyroid-stimulating hormone (TSH), Free T4, and insulin were measured by Cobas 8000 e602 (Roche, Mannheim, Germany) using each reagent. The radioimmunoassay (RIA) method was used to analyze free testosterone using 1470 Wizard Y-counter (PerkinElmer, Turku, Finland) with a free testosterone RIA CT kit (Asbach Medical Products, Obrigheim, Germany). Insulin-like growth factor (IGF-1) and dehydroepiandrosterone (DHEA) were measured by a microplate reader (VERSAmax, Sunnyvale, USA) with an IGF-1 enzyme-linked immunosorbent assay (ELISA) kit (Mediagnost, Reutlingen, Germany) and DHEA ELISA kit (IBL International, Hamburg, Germany).

\subsection{Ethical Considerations}

The KFACS was approved by the institutional review board (IRB) at the Kyung Hee Medical Center, Seoul, Republic of Korea (KHUH 2015-12-103). As a secondary analysis using de-identified data, this study was exempt from IRB review (KHSIRB-20-234).

\subsection{Statistical Analyses}

The G*Power (version 3.1.9.5., University of Dusseldorf, Dusseldorf, Germany) software was used to calculate the sample size for this study. The expected correlation between clinical blood markers and HRQoL was 0.1. With an alpha value of 0.05 and a power of 0.95 , we calculated the estimated sample size to be 1077 [28]. All statistical analyses were performed using SPSS version 25.0 (IBM SPSS Statistics, SPSS, Chicago, IL, USA). A $p$-value of $\leq 0.05$ was considered statistically significant. All data were presented as the mean (standard deviation (SD)) and percentage. Hierarchical cluster analysis was used to identify the subgroups of symptom clusters. Hierarchical cluster analysis was performed 
using Ward's method on squared Euclidian distances using the four symptoms: pain, mobility limitation, fatigue, and sleep deprivation. The appropriate number of clusters was selected using the agglomeration schedule. The subgroups were compared to demographic characteristics using the chi-square test, one-way analysis of variance (ANOVA) and the Kruskal-Wallis test. We performed logistic regression to identify the associated factors, including socio-demographics and the presence of chronic conditions, on the likelihood of a high symptom burden. Data were expressed as odds ratios (ORs) with $95 \%$ confidence intervals (CIs). We performed Spearman's rank correlation analysis to explore the relationships between the number of symptoms, HRQoL, and biomarkers.

\section{Results}

\subsection{General Characteristics of Participants}

A total of 2362 participants who met the inclusion criteria were included in this analysis. More than $55 \%$ of the samples were females with a mean age of 76.1 (3.9) and 8.55 (5.72) of education. The majority of the participants were married (66\%), living with others $(76 \%)$, and residing in an urban area (73\%). More than $70 \%$ were overweight (BMI $\geq 23.0)$. Participants reported that they had light physical activity more than 5 days/week on average. More than $80 \%$ of the participants were diagnosed with cardiovascular disease. Nearly $40 \%$ of the participants had a musculoskeletal disease. The participants who were diagnosed with cerebrovascular, respiratory, gastrointestinal, neurological disease, and cancer were less than $10 \%$. Nearly $60 \%$ were living with at least two chronic illnesses (Table 1 ).

Table 1. Demographics and general characteristics of the symptom cluster subgroups.

\begin{tabular}{|c|c|c|c|c|c|c|}
\hline \multirow{2}{*}{ Variables } & \multirow{2}{*}{ Categories } & \multirow{2}{*}{$\begin{array}{c}\text { Total }(n=2362) \\
\mathrm{M}(\mathrm{SD}) / n(\%)\end{array}$} & \multirow{2}{*}{$\begin{array}{c}\text { HSB }(n=1032) \\
\text { M(SD) } / n(\%)\end{array}$} & \multirow{2}{*}{$\begin{array}{l}\text { PAF }(n=566) \\
\mathrm{M}(\mathrm{SD}) / n(\%)\end{array}$} & \multirow{2}{*}{$\begin{array}{l}\mathrm{SDP}(n=764) \\
\mathrm{M}(\mathrm{SD}) / n(\%)\end{array}$} & \multirow{2}{*}{$p$} \\
\hline & & & & & & \\
\hline Age (years) ${ }^{\dagger}$ & & $76.1(3.9)$ & $76.9(3.9)$ & $75.6(3.8)$ & $75.3(3.67)$ & $<0.001$ \\
\hline \multirow{3}{*}{ Sex } & & & & & & $<0.001$ \\
\hline & Males & 1053 (44.6) & $261(25.3)$ & $326(57.6)$ & $466(61.0)$ & \\
\hline & Females & $1309(55.4)$ & $771(74.7)$ & $240(42.4)$ & $298(39.0)$ & \\
\hline \multirow{4}{*}{ Body mass index } & & & & & & 0.004 \\
\hline & Underweight & $36(1.5)$ & $20(1.9)$ & $10(1.8)$ & $6(0.8)$ & \\
\hline & Normal & $654(27.7)$ & $250(24.2)$ & $178(31.4)$ & $226(29.6)$ & \\
\hline & Overweight & $1672(70.8)$ & $762(73.8)$ & $378(66.8)$ & $532(70.8)$ & \\
\hline \multirow{3}{*}{ Smoking } & & & & & & $<0.001$ \\
\hline & Ever & $851(36.1)$ & $214(20.8)$ & $259(45.8)$ & $378(49.6)$ & \\
\hline & Never & 1507 (63.9) & $816(79.2)$ & $307(54.2)$ & $384(50.4)$ & \\
\hline \multirow{3}{*}{ Alcohol consumption } & & & & & & $<0.001$ \\
\hline & Ever & $1646(69.8)$ & 635 (61.7) & $419(74.2)$ & $592(77.6)$ & \\
\hline & Never & $712(30.2)$ & $395(38.3)$ & $146(23.9)$ & $171(22.4)$ & \\
\hline \multirow{3}{*}{$\begin{array}{c}\text { Physical activity }{ }^{\dagger} \text { (days } \\
\text { per week) }\end{array}$} & Vigorous & $0.31(1.13)$ & $0.17(0.88)$ & $0.37(1.25)$ & $0.45(1.30)$ & $<0.001$ \\
\hline & Moderate & $2.51(2.61)$ & $2.10(2.50)$ & $2.74(2.60)$ & $2.89(2.69)$ & $<0.001$ \\
\hline & Light & $5.36(2.29)$ & $4.98(2.51)$ & $5.51(2.15)$ & $5.77(1.99)$ & $<0.001$ \\
\hline Education (years) $^{\dagger}$ & & $8.55(5.72)$ & $6.70(6.36)$ & $9.59(4.68)$ & $10.27(4.73)$ & $<0.001$ \\
\hline \multirow{3}{*}{ Marital status } & & & & & & $<0.001$ \\
\hline & Married & $1547(65.6)$ & $536(52.0)$ & $426(75.3)$ & $585(76.7)$ & \\
\hline & $\begin{array}{l}\text { Divorced and } \\
\text { widowed }\end{array}$ & $813(34.4)$ & $495(48.0)$ & $140(24.7)$ & $178(23.3)$ & \\
\hline \multirow{3}{*}{ Living status } & & & & & & $<0.001$ \\
\hline & With others & $1803(76.3)$ & $691(67.0)$ & $464(82.0)$ & $648(84.8)$ & \\
\hline & Alone & $559(23.7)$ & $341(33.0)$ & $102(18.0)$ & $116(15.2)$ & \\
\hline \multirow{3}{*}{ Living area } & & & & & & $<0.001$ \\
\hline & Urban & $1724(73.4)$ & $711(69.2)$ & $400(70.9)$ & $613(80.8)$ & \\
\hline & Rural & $626(26.6)$ & $316(30.8)$ & $164(29.1)$ & $146(19.2)$ & \\
\hline \multirow{3}{*}{ Home ownership } & & & & & & $<0.001$ \\
\hline & Owner occupied & $1893(80.2)$ & 771 (74.9) & $478(84.5)$ & $644(84.3)$ & \\
\hline & Rented & 466 (19.7) & $258(25.1)$ & $88(15.5)$ & $120(15.7)$ & \\
\hline
\end{tabular}


Table 1. Conts.

\begin{tabular}{|c|c|c|c|c|c|c|}
\hline \multirow{2}{*}{ Variables } & \multirow{2}{*}{ Categories } & \multirow{2}{*}{$\begin{array}{c}\text { Total }(n=2362) \\
\mathrm{M}(\mathrm{SD}) / n(\%)\end{array}$} & \multirow{2}{*}{$\begin{array}{c}\text { HSB }(n=1032) \\
\mathrm{M}(\mathrm{SD}) / n(\%)\end{array}$} & \multirow{2}{*}{$\begin{array}{l}\text { PAF }(n=566) \\
\mathrm{M}(\mathrm{SD}) / n(\%)\end{array}$} & \multirow{2}{*}{$\begin{array}{c}\mathrm{SDP}(n=764) \\
\mathrm{M}(\mathrm{SD}) / n(\%)\end{array}$} & \multirow{2}{*}{$p$} \\
\hline & & & & & & \\
\hline \multirow{2}{*}{ Cardiovascular disease } & Yes & 1934 (81.9) & 842 (81.6) & 465 (82.2) & $627(82.1)$ & 0.948 \\
\hline & No & $428(18.1)$ & $190(18.4)$ & $101(17.8)$ & $137(17.9)$ & \\
\hline \multirow{3}{*}{ Cerebrovascular disease } & & & & & & 0.864 \\
\hline & Yes & $135(5.7)$ & $56(5.4)$ & $34(6.0)$ & $45(5.9)$ & \\
\hline & No & $2227(94.3)$ & $976(94.6)$ & $532(94.0)$ & $719(94.1)$ & \\
\hline \multirow{3}{*}{ Musculoskeletal disease } & & & & & & $<0.001$ \\
\hline & Yes & $992(42.0)$ & $592(57.4)$ & $188(33.2)$ & $212(27.7)$ & \\
\hline & No & $1370(58.0)$ & $440(42.6)$ & $378(66.8)$ & $552(72.3)$ & \\
\hline \multirow{3}{*}{ Respiratory disease } & & & & & & 0.417 \\
\hline & Yes & 179 (7.6) & $86(8.3)$ & $42(7.4)$ & $51(6.7)$ & \\
\hline & No & $2183(92.4)$ & $946(91.7)$ & $524(92.6)$ & $713(93.3)$ & \\
\hline \multirow{3}{*}{ Gastrointestinal disease } & & & & & & $<0.001$ \\
\hline & Yes & $190(8.0)$ & $107(10.4)$ & $49(8.7)$ & $34(4.5)$ & \\
\hline & No & $2172(92.0)$ & $925(89.6)$ & $517(91.3)$ & $730(95.5)$ & \\
\hline \multirow{3}{*}{ Neurological disease } & & & & & & 0.407 \\
\hline & Yes & $21(0.9)$ & $11(1.1)$ & $5(0.9)$ & $5(0.7)$ & \\
\hline & No & $2341(99.1)$ & $1021(98.9)$ & $561(99.1)$ & $759(99.3)$ & \\
\hline \multirow{3}{*}{ Renal disease } & & & & & & 0.070 \\
\hline & Yes & $44(1.9)$ & $15(1.5)$ & $17(3.0)$ & $12(1.6)$ & \\
\hline & No & $2318(98.1)$ & $1017(98.5)$ & $549(97.0)$ & $752(98.4)$ & \\
\hline \multirow{3}{*}{ Endocrine disease } & & & & & & 0.444 \\
\hline & Yes & $712(30.1)$ & $297(28.8)$ & 177 (31.3) & $238(31.2)$ & \\
\hline & No & $1650(69.9)$ & 735 (71.2) & 389 (68.7) & $526(68.8)$ & \\
\hline \multirow{3}{*}{ Cancer } & & & & & & 0.257 \\
\hline & Yes & $91(3.9)$ & $33(3.2)$ & $22(3.9)$ & $36(4.7)$ & \\
\hline & No & $2271(96.1)$ & 999 (96.8) & $544(96.1)$ & $728(95.3)$ & \\
\hline \multirow{2}{*}{$\begin{array}{c}\text { Number of chronic } \\
\text { illnesses }\end{array}$} & & & & & & $<0.001$ \\
\hline & $\begin{array}{c}1 \\
\geq 2\end{array}$ & $\begin{array}{c}979(41.4) \\
1383(58.6)\end{array}$ & $\begin{array}{l}336(32.6) \\
696(67.4)\end{array}$ & $\begin{array}{l}256(45.2) \\
310(54.8)\end{array}$ & $\begin{array}{l}387(50.7) \\
377(49.3)\end{array}$ & \\
\hline
\end{tabular}

Note: HSB, high symptom burden; PAF, pain and fatigue; SDP, sleep deprivation; data were presented as the mean (standard deviation (SD)) or a percentage. The $p$-values were based on the chi-square test, one-way analysis of variance (ANOVA), and the Kruskal-Wallis test ${ }^{\dagger}$.

\subsection{Description of Symptom Clusters}

The cluster analysis of the symptoms (pain, mobility limitation, fatigue, and sleep deprivation) showed three symptom cluster profiles: subgroup 1, high symptom burden (HSB) group ( $n=1032,43.7 \%)$; subgroup 2, pain and fatigue (PAF) group $(n=566,24.0 \%)$; and subgroup 3, sleep deprivation (SDP) group ( $n=764,32.3 \%$ ) (Table 2). Among the three subgroups, participants in the PAF and SDP subgroups were younger $(p<0.001)$, reported a higher number of days of physical activity $(p<0.001)$, and had a higher proportion of participants with only one chronic condition than the HSB subgroup $(p<0.001)$ (Table 1$)$.

Table 2. Classification of symptoms in the symptom cluster subgroups.

\begin{tabular}{|c|c|c|c|c|c|}
\hline Variables & Total & HSB & PAF & SDP & $p$ \\
\hline Total number of participants & 2362 & $1032(43.7 \%)$ & $566(24.0 \%)$ & $764(32.3 \%)$ & \\
\hline Pain & 1191 & $793(66.6 \%)$ & $398(33.4 \%)$ & $0(0.0 \%)$ & $<0.001$ \\
\hline Mobility limitation & 773 & $773(100 \%)$ & $0(0.0 \%)$ & $0(0.0 \%)$ & $<0.001$ \\
\hline Fatigue & 959 & $649(67.7 \%)$ & $310(32.3 \%)$ & $0(0.0 \%)$ & $<0.001$ \\
\hline Sleep deprivation & 834 & $583(69.9 \%)$ & $0(0.0 \%)$ & $251(30.1 \%)$ & $<0.001$ \\
\hline
\end{tabular}

Note: HSB, high symptom burden; PAF, pain and fatigue; SDP, sleep deprivation. 


\subsection{Predicting Variables with High Symptom Burden}

We selected the potential associated factors that were significantly different between groups. The full model containing all predictors was statistically significant, $\chi^{2}(14$, $n=2362)=569.05(p<0.001)$. The model that explained the Cox and Snell R square was $22 \%$, and Nagelkerke R squared was $29 \%$. We found that the following were significant contributors to the model: age ( $\mathrm{OR}=1.11)$, being female $(\mathrm{OR}=2.40)$, $\mathrm{BMI}(\mathrm{OR}=1.05)$, non-smoker $(\mathrm{OR}=1.51)$, moderate physical activity $(\mathrm{OR}=0.95)$, light physical activity $(\mathrm{OR}=0.89)$, years of education $(\mathrm{OR}=0.93)$, living in a rural area $(\mathrm{OR}=1.36)$, and living with chronic illnesses for more than 2 years $(\mathrm{OR}=1.52)$ (Table 3$)$. Sex was the strongest predictor of high symptom burden, recording an odds ratio of 2.40. This result indicated that females were over 2.40 times more likely to have a high symptom burden than men, controlling for all other factors in the model.

Table 3. Logistic regression predicting the likelihood of high symptom burden.

\begin{tabular}{cccc}
\hline Variables & OR & $\mathbf{9 5 \%}$ CI & $p$ \\
\hline Age & 1.113 & $1.085-1.142$ & $<0.001$ \\
Female & 2.398 & $1.715-3.354$ & $<0.001$ \\
Body mass index & 1.049 & $1.016-1.083$ & 0.003 \\
Non smoker & 1.508 & $1.094-2.079$ & 0.012 \\
Non alcohol drinker & 1.035 & $0.835-1.284$ & 0.751 \\
Days of vigorous physical activity & 0.911 & $0.829-1.001$ & 0.054 \\
Days of moderate physical activity & 0.952 & $0.917-0.988$ & 0.009 \\
Days of light physical activity & 0.886 & $0.850-0.924$ & $<0.001$ \\
Rural & 1.356 & $1.083-1.698$ & 0.008 \\
Yeas of education & 0.932 & $0.911-0.952$ & $<0.001$ \\
Divorced and widowed & 1.024 & $0.753-1.392$ & 0.882 \\
Alone & 1.170 & $0.848-1.614$ & 0.339 \\
Rented & 1.210 & $0.947-1.547$ & 0.128 \\
Number of chronic illnesses $\geq 2$ & 1.517 & $1.251-1.839$ & $<0.001$ \\
\hline Note: OR, odds ratio; CI, confidence interval & & &
\end{tabular}

Note: OR, odds ratio; CI, confidence interval.

\subsection{Correlations of Health-Related Quality of Life and Clinical Blood Markers among Different Symptom Clusters}

The results of the present study show the unique associations of clinical blood markers and HRQoL among these three symptom cluster subgroups (Table 4). Although no significant associations among HRQoL and clinical blood markers were shown in the PAF cluster, the insulin, free testosterone, and DHEA levels were significantly associated with either physical health or mental health in the HSB and SDP clusters. In the HSB cluster, physical health was negatively associated with free T4 $(r=-0.083, p<0.01)$ and insulin $(r=-0.084 . p<0.01)$ levels. In the SDP cluster, physical health was negatively associated with WBC $(r=-0.086, p<0.05)$ and insulin $(r=-0.111, p<0.01)$.

We also found that the relationships among sex-specific blood markers and HRQoL components were different among the three cluster groups. While there were no significant correlations of the clinical blood makers and HRQoL in the PAF group, in the HSB group, low free testosterone (male: $r=0.124$, female: $r=0.110, p<0.05$ ) and low DHEA (male: $r=0.352$ and female: $r=0.134, p<0.05$ ) were associated with low physical health. In the SDP group, free testosterone was significantly associated only with the mental health component of HRQoL for both male $(r=-0.093, p<0.05)$ and female $(r=-0.116, p<0.05)$ participants (Table 5). 
Table 4. Associations of symptom clusters, health-related quality of life, and clinical blood markers.

\begin{tabular}{|c|c|c|c|c|c|c|c|c|c|c|}
\hline \multirow{2}{*}{ Subgroups } & \multirow{2}{*}{ Variables } & \multirow{2}{*}{ Mean (SD) } & \multicolumn{8}{|c|}{ Spearman's Rho } \\
\hline & & & PCS-12 & MCS-12 & WBC & hs-CRP & TSH & Free $\mathrm{T} 4$ & Insulin & IGF-1 \\
\hline \multirow{7}{*}{$\begin{array}{l}\text { High } \\
\text { symptoms } \\
\text { burden }\end{array}$} & 1. PCS-12 & $35.33(9.89)$ & 1 & $0.093^{* *}$ & -0.049 & -0.038 & 0.043 & $-0.083^{* *}$ & $-0.084^{* *}$ & 0.052 \\
\hline & 2. MCS-12 & 49.18 (11.63) & & 1 & 0.025 & 0.055 & 0.014 & 0.032 & 0.011 & -0.063 \\
\hline & 3. WBC $(1000 / \mu \mathrm{L})$ & $5.98(1.77)$ & & & 1 & $0.203^{* *}$ & $-0.068 *$ & $0.113^{* *}$ & $0.214^{* *}$ & 0.083 \\
\hline & 4. hs-CRP (mg/L) & $1.39(1.92)$ & & & & 1 & -0.02 & -0.01 & $0.089^{* *}$ & 0.02 \\
\hline & 5. TSH (uIU/mL) & $2.80(5.31)$ & & & & & 1 & $-0.300^{* *}$ & -0.012 & -0.069 \\
\hline & 6. Free T4 (ng/dL) & $1.23(0.19)$ & & & & & & 1 & -0.061 & -0.008 \\
\hline & 8. IGF-1 (ng/mL) & $108.37(58.52)$ & & & & & & & & 1 \\
\hline \multirow{8}{*}{$\begin{array}{l}\text { Pain and } \\
\text { fatigue }\end{array}$} & 1. PCS-12 & $44.99(8.53)$ & 1 & -0.006 & -0.033 & -0.011 & 0.029 & -0.045 & -0.036 & -0.005 \\
\hline & 2. MCS-12 & $52.98(9.09)$ & & 1 & 0.047 & 0.003 & -0.057 & 0.04 & 0.027 & 0.075 \\
\hline & 3. WBC $(1000 / \mu \mathrm{L})$ & $6.05(1.53)$ & & & 1 & $0.191^{* *}$ & -0.079 & $0.159 * *$ & $0.202^{* *}$ & 0.014 \\
\hline & 4. hs-CRP (mg/L) & $1.39(2.23)$ & & & & 1 & $0.087 *$ & -0.068 & 0.004 & -0.057 \\
\hline & 5. TSH (uIU/mL) & $2.71(4.43)$ & & & & & 1 & $-0.253^{* *}$ & -0.05 & -0.14 \\
\hline & 6. Free T4 (ng/dL) & $1.24(0.21)$ & & & & & & 1 & $-0.125^{* *}$ & 0.135 \\
\hline & $\begin{array}{l}\text { 7. Insulin } \\
\text { (uU/mL) }\end{array}$ & $8.08(9.34)$ & & & & & & & 1 & 0.021 \\
\hline & 8. IGF-1 (ng/mL) & $123.68(68.35)$ & & & & & & & & 1 \\
\hline \multirow{8}{*}{$\begin{array}{c}\text { Sleep } \\
\text { deprivation }\end{array}$} & 1. PCS-12 & $49.73(6.53)$ & 1 & $-0.185^{* *}$ & -0.016 & $-0.086^{*}$ & 0.025 & 0.005 & $-0.111^{* *}$ & 0.077 \\
\hline & 2. MCS-12 & $56.09(7.13)$ & & 1 & -0.022 & 0.048 & 0.025 & -0.019 & 0.037 & -0.114 \\
\hline & 3. WBC $(1000 / \mu \mathrm{L})$ & $5.83(1.90)$ & & & 1 & $0.175^{* *}$ & 0.012 & 0.079 * & $0.197^{* *}$ & 0.049 \\
\hline & 4. hs-CRP (mg/L) & $1.35(2.07)$ & & & & 1 & 0.036 & -0.011 & $0.147^{* *}$ & -0.039 \\
\hline & 5. TSH (uIU/mL) & $2.68(2.54)$ & & & & & 1 & $-0.334^{* *}$ & -0.039 & -0.065 \\
\hline & 6. Free T4 (ng/dL) & $1.23(0.20)$ & & & & & & 1 & $-0.085^{*}$ & 0.044 \\
\hline & $\begin{array}{l}\text { 7. Insulin } \\
(\mathrm{uU} / \mathrm{mL})\end{array}$ & $7.67(9.02)$ & & & & & & & 1 & -0.029 \\
\hline & 8. IGF-1 (ng/mL) & $123.13(66.12)$ & & & & & & & & 1 \\
\hline
\end{tabular}

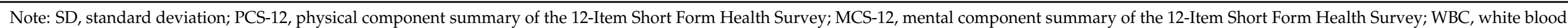
Note: SD, standard deviation; PCS-12, physical component summary of the 12-Item Short Form Health Survey; MCS-12, mental component su
cell; hs-CRP, high sensitivity C reactive protein; TSH, thyroid stimulating hormone; IGF-1, insulin-like growth factor I; $* p<0.05 ; * * p<0.01$. 
Table 5. Associations of symptoms clusters, health-related quality of life, and sex-specific clinical blood markers.

\begin{tabular}{|c|c|c|c|c|c|c|c|}
\hline \multirow{2}{*}{ Subgroups } & \multirow{2}{*}{ Sex } & \multirow{2}{*}{ Variables } & \multirow{2}{*}{ Mean (SD) } & \multicolumn{4}{|c|}{ Spearman's Rho } \\
\hline & & & & PCS-12 & MCS-12 & Free Testosterone & DHEA \\
\hline \multirow{7}{*}{$\begin{array}{l}\text { High symptoms } \\
\text { burden }\end{array}$} & \multirow{4}{*}{ Male } & 1. PCS-12 & $37.77(10.62)$ & \multirow[t]{4}{*}{1} & \multirow{4}{*}{$\begin{array}{c}0.008 \\
1\end{array}$} & \multirow{4}{*}{$\begin{array}{c}0.124 * \\
0.077 \\
1\end{array}$} & $0.352 * *$ \\
\hline & & 2. MCS-12 & $50.59(10.90)$ & & & & -0.097 \\
\hline & & 3. Free testosterone $(\mathrm{pg} / \mathrm{mL})$ & $8.85(3.61)$ & & & & $0.510 * *$ \\
\hline & & 4. DHEA $(\mathrm{ng} / \mathrm{mL})$ & $0.86(0.57)$ & & & & 1 \\
\hline & \multirow{3}{*}{ Female } & 1. PCS-12 & $34.51(9.50)$ & \multirow[t]{3}{*}{1} & \multirow{3}{*}{$\begin{array}{c}0.107^{* *} \\
1\end{array}$} & \multirow{3}{*}{$\begin{array}{c}0.110^{* *} \\
0.062 \\
1\end{array}$} & $0.134 *$ \\
\hline & & 2. MCS-12 & $48.70(11.83)$ & & & & -0.037 \\
\hline & & 4. DHEA $(\mathrm{ng} / \mathrm{mL})$ & $0.75(0.58)$ & & & & 1 \\
\hline \multirow{7}{*}{ Pain and fatigue } & \multirow{4}{*}{ Male } & 1. PCS-12 & $45.09(8.70)$ & \multirow[t]{4}{*}{1} & 0.018 & \multirow{4}{*}{$\begin{array}{c}0.031 \\
-0.075 \\
1\end{array}$} & 0.007 \\
\hline & & 2. MCS-12 & $52.82(9.40)$ & & 1 & & -0.077 \\
\hline & & 3. Free testosterone $(\mathrm{pg} / \mathrm{mL})$ & $9.06(3.29)$ & & & & $0.384^{* *}$ \\
\hline & & 4. DHEA $(\mathrm{ng} / \mathrm{mL})$ & $0.99(1.03)$ & & & & 1 \\
\hline & \multirow{3}{*}{ Female } & 1. PCS-12 & $44.86(8.32)$ & \multirow[t]{3}{*}{1} & -0.038 & \multirow{3}{*}{$\begin{array}{c}0.004 \\
0.02 \\
1\end{array}$} & 0.155 \\
\hline & & 2. MCS-12 & $53.21(8.67)$ & & 1 & & 0.01 \\
\hline & & 3. Free testosterone $(\mathrm{pg} / \mathrm{mL})$ & $0.96(1.00)$ & & & & $0.659 * *$ \\
\hline \multirow{8}{*}{ Sleep deprivation } & \multirow{4}{*}{ Male } & 1. PCS-12 & $50.50(6.11)$ & \multirow[t]{4}{*}{1} & $-0.182^{* *}$ & \multirow{4}{*}{$\begin{array}{c}0.063 \\
-0.093 * \\
1\end{array}$} & 0.066 \\
\hline & & 2. MCS-12 & $56.50(6.26)$ & & 1 & & $-0.161 *$ \\
\hline & & 3. Free testosterone $(\mathrm{pg} / \mathrm{mL})$ & $9.33(3.29)$ & & & & $0.381 * *$ \\
\hline & & 4. DHEA (ng/mL) & $0.98(0.67)$ & & & & 1 \\
\hline & \multirow{4}{*}{ Female } & 1. PCS-12 & $48.52(6.98)$ & \multirow[t]{4}{*}{1} & $-0.194^{* *}$ & \multirow{4}{*}{$\begin{array}{c}0.102 \\
-0.116 \\
1\end{array}$ * } & -0.021 \\
\hline & & 2. MCS-12 & $55.45(8.29)$ & & 1 & & -0.155 \\
\hline & & 3. Free testosterone $(\mathrm{pg} / \mathrm{mL})$ & $0.94(0.89)$ & & & & $0.580^{* *}$ \\
\hline & & 4. DHEA $(\mathrm{ng} / \mathrm{mL})$ & $1.00(1.16)$ & & & & 1 \\
\hline
\end{tabular}

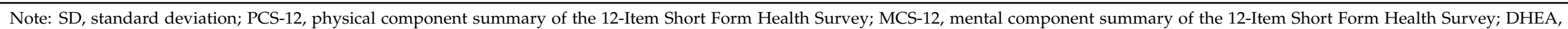

dehydroepiandrosterone; ${ }^{*} p<0.05 ;{ }^{* *} p<0.01$. 


\section{Discussion}

Among the Korean community-dwelling older adults, we found three clusters with different symptom compositions, including pain, mobility limitation, fatigue, and sleep deprivation. Pain, fatigue, mobility problems, and sleep deprivation are common in most chronic conditions, such as chronic obstructive pulmonary disease, chronic kidney disease, and lung cancer [7,29-31]. Our study provides a unique perspective on the subgroups of these symptoms including the high symptoms burden subgroup (HSB: $44 \%$ of our participants), the pain and fatigue subgroup (PAF: 24\%), and the sleep deprivation subgroup (SDP: $32 \%$ ).

Similar to other studies which enrolled participants with various chronic conditions such as end-stage renal disease, atrial fibrillation, and lung cancer [32-36], we found that age, sex, and having multiple chronic conditions were associated with high symptom burden and poor HRQoL. We also found that more than $24 \%$ of our participants mainly suffered from pain and fatigue (PAF subgroup) and that $32 \%$ mainly suffered from sleep deprivation (SDP subgroup). The participants in these two subgroups (PAF and SDP) were younger, reported a higher number of days engaged in physical activity, and were more likely having only one chronic condition than the HSB subgroup. Consistently with our findings, a national survey of community-dwelling older adults in the United States showed that older adults who suffered from one or two symptoms were younger and had a small number of chronic conditions than the over three symptoms group [37]. This finding might introduce symptoms profiling as an alternative way to tailor interventions such as exercise programs for people who may have multiple chronic conditions. Age, active lifestyle and the number of underlying chronic conditions were significant predictors of the success of many rehabilitation programs [38,39]; however, tailored programs based on age, lifestyle and the number of chronic conditions can be challenging. Thus, we suggest that a tailored intervention program is needed to efficiently manage symptoms for older adults living with chronic conditions.

Studies have suggested sex differences in symptoms experience [40-42]. We found that sex was the strongest predictor of high symptom burden. Females were two-fold more likely to experience a high symptom burden than males. Other studies among older adults in Japan [43], Singapore [44], and South Korea [45-47] reported that females have higher levels of symptoms (e.g., pain, fatigue, depression and sleep disturbance) than their male counterparts. The mechanism of higher symptoms burden in females is unclear. Studies have suggested that the role of gonadal sex steroids (e.g., estrogen, progesterone and testosterone) levels may influence their symptoms experiences [48-50]. Our study found that high sex hormones (free testosterone and DHEA) were significantly associated with the high HRQoL physical component in both the HSB and SDP subgroups but not in the PAF subgroup. These associations were significant even after we divided the subgroups based on sex. Our finding provides additional evidence to support the role of testosterone, a male sex hormone, in maintaining physical function and the perceived quality of life [50,51].

The DHEA hormone is an essential precursor for the production of female sex hormones - estrogen and androgen [52] — and has long been known for its ability to improve depressive symptoms [53]. Another study found the significant correlation between low DHEA and poor physical function [19]. Our study found that high DHEA levels were significantly associated with the high HRQoL-physical component, though only in the HSB subgroup for both male and female participants. Only the SDP subgroup showed a significant correlation between high DHEA levels and low HRQoL-mental component, but only among male participants. No significant correlation between DHEA and HRQoL was found in the PAF subgroup. These results suggest the unique role of DHEA in the symptoms experienced between the sexes, which may explain why people respond to DHEA supplementation differently. DHEA supplementation increased the plasma level of testosterone in both males and females, but the prominent increase was shown in female, healthier and younger participants [54,55]. 
We acknowledge the number of limitations of our study, which include: (a) the crosssectional nature of the study; (b) the symptoms (pain, fatigue, and sleep deprivation) being measured by single-item and dichotomous questions; (c) the homogenous population that may only be generalized to the Korean community-dwelling older adults; (d) patients with acute stroke or myocardial infarction during the past six months, or systolic blood pressure over $180 \mathrm{mmHg}$ were excluded due to the parent study exclusion criteria; and (e) only inflammatory and endocrine biomarkers were included in the analysis. However, our study provides an initial glimpse of various symptom cluster profiles that have an impact on HRQoL, as influenced by the clinical blood markers collected from a large nationwide sample size $(n=2362)$. Our study provides a unique perspective on predictive factors for high symptom burden and the roles of sex-specific hormones among older adults. Further studies are required to further explore the relationships revealed in this study.

\section{Conclusions}

Korean community-dwelling older adults living with chronic conditions suffer from various symptoms that reduce their HRQoL: pain, limited mobility, fatigue, and sleep deprivation. Our findings suggested the potential role of sex and sex hormones such as DHEA in the symptoms of Korean community-dwelling older adults. In terms of symptom management, understanding unique symptom cluster profiles and their predictive markers is worthwhile to develop optimal and precise interventions.

Author Contributions: Conceptualization, J.P. and N.L.; methodology, J.P., Y.K. and N.P.; software, J.P. and N.P.; validation, J.P., N.L., L.N.S. and C.W.W.; formal analysis, J.P.; investigation, J.P. and C.W.W.; resources, C.W.W.; data curation, J.P., N.L. and N.P.; writing-original draft preparation, J.P. and Y.K.; writing-review and editing, N.L., L.N.S.; visualization, J.P.; supervision, N.L. and C.W.W.; project administration, C.W.W.; funding acquisition, J.P. and C.W.W. All authors have read and agreed to the published version of the manuscript.

Funding: This research was funded by a grant from the Korea Health Technology R\&D Project through the Korean Health Industry Development Institute, funded by the Ministry of Health and Welfare, Republic of Korea (grant number: HI15C3153) and National Research Foundation of Korea funded by the Ministry of Education (grant number: 2019R1C1C1005519).

Institutional Review Board Statement: The study was conducted according to the guidelines of the Declaration of Helsinki and approved by the institutional review board (IRB) at the Kyung Hee Medical Center, Seoul, Republic of Korea (KHUH 2015-12-103). As a secondary analysis using de-identified data, this study was exempt from IRB review (KHSIRB-20-234).

Informed Consent Statement: Not applicable.

Data Availability Statement: Data sharing is not applicable to this article.

Conflicts of Interest: The authors declare no conflict of interest.

\section{References}

1. United Nations, Department of Economic and Social Affairs, Population Division. Population Ageing 2019: Highlights; United Nations: New York, NY, USA, 2019.

2. Kim, Y.I.; Kim, Y.Y.; Yoon, J.L.; Won, C.W.; Ha, S.; Cho, K.D.; Park, B.R.; Bae, S.; Lee, E.J.; Park, S.Y.; et al. Cohort Profile: National health insurance service-senior (NHIS-senior) cohort in Korea. BMJ Open 2019, 9, e024344. [CrossRef] [PubMed]

3. Lee, J.; Jang, S.N.; Cho, S.I. The Trajectories of the Number of Pain Sites and Their Associated Factors in Older Adults: Results from the Korean Longitudinal Study of Ageing. Gerontology 2018, 64, 532-540. [CrossRef] [PubMed]

4. Cao, X.; Chen, Z.; Wu, L.; Zhou, J. Co-occurrence of chronic pain, depressive symptoms, and poor sleep quality in a health check-up population in China: A multicenter survey. J. Affect. Disord. 2020, 281, 792-798. [CrossRef] [PubMed]

5. Yesilbalkan, O.U.; Ozkaraman, A.; Soydinc, T. Comparing the symptoms experience of cancer and non-cancer older adult patients in Turkish cancer patients: A cross-sectional study. JPMA 2020, 70, 147-150. [CrossRef]

6. Lee, S.J.; Jeon, J. Relationship between symptom clusters and quality of life in patients at stages 2 to 4 chronic kidney disease in Korea. Appl. Nurs. Res. 2015, 28, e13-e19. [CrossRef]

7. Lim, K.E.; Kim, S.R.; Kim, H.K.; Kim, S.R. Symptom Clusters and Quality of Life in Subjects With COPD. Respir. Care 2017, 62, 1203-1211. [CrossRef] [PubMed] 
8. Henchoz, Y.; Bula, C.; Guessous, I.; Rodondi, N.; Goy, R.; Demont, M.; Santos-Eggimann, B. Chronic symptoms in a representative sample of community-dwelling older people: A cross-sectional study in Switzerland. BMJ Open 2017, 7, e014485. [CrossRef]

9. Salanitro, A.H.; Hovater, M.; Hearld, K.R.; Roth, D.L.; Sawyer, P.; Locher, J.L.; Bodner, E.; Brown, C.J.; Allman, R.M.; Ritchie, C.S. Symptom burden predicts hospitalization independent of comorbidity in community-dwelling older adults. J. Am. Geriatr. Soc. 2012, 60, 1632-1637. [CrossRef]

10. Sheppard, K.D.; Brown, C.J.; Hearld, K.R.; Roth, D.L.; Sawyer, P.; Locher, J.L.; Allman, R.M.; Ritchie, C.S. Symptom burden predicts nursing home admissions among older adults. J. Pain Symptom Manag. 2013, 46, 591-597. [CrossRef]

11. Portz, J.D.; Kutner, J.S.; Blatchford, P.J.; Ritchie, C.S. High symptom burden and low functional status in the setting of multimorbidity. J. Am. Geriatr. Soc. 2017, 65, 2285-2289. [CrossRef]

12. Zisberg, A.; Shulyaev, K.; Nurit, G.; Agmon, M.; Dorit, P. Symptom clusters in hospitalized older adults: Characteristics and outcomes. Geriatr. Nurs. 2020. [CrossRef] [PubMed]

13. Miaskowski, C. Future Directions in Symptom Cluster Research; Seminars in oncology nursing; Elsevier: Amsterdam, The Netherlands, 2016; Volume 32, pp. 405-415.

14. Kim, H.; McGuire, D.B.; Tulman, L.; Barsevick, A.M. Symptom clusters: Concept analysis and clinical implications for cancer nursing. Cancer Nurs. 2005, 28, 270-282. [CrossRef] [PubMed]

15. Choghakhori, R.; Abbasnezhad, A.; Hasanvand, A.; Amani, R. Inflammatory cytokines and oxidative stress biomarkers in irritable bowel syndrome: Association with digestive symptoms and quality of life. Cytokine 2017, 93, 34-43. [CrossRef]

16. Colbert, L.H.; Visser, M.; Simonsick, E.M.; Tracy, R.P.; Newman, A.B.; Kritchevsky, S.B.; Pahor, M.; Taaffe, D.R.; Brach, J.; Rubin, S. Physical activity, exercise, and inflammatory markers in older adults: Findings from the Health, Aging and Body Composition Study. J. Am. Geriatr. Soc. 2004, 52, 1098-1104. [CrossRef]

17. Jackowska, M.; Kumari, M.; Steptoe, A. Sleep and biomarkers in the English Longitudinal Study of Ageing: Associations with C-reactive protein, fibrinogen, dehydroepiandrosterone sulfate and hemoglobin. Psychoneuroendocrinology 2013, 38, 1484-1493. [CrossRef]

18. Penninx, B.W.; Kritchevsky, S.B.; Newman, A.B.; Nicklas, B.J.; Simonsick, E.M.; Rubin, S.; Nevitt, M.; Visser, M.; Harris, T.; Pahor, M. Inflammatory markers and incident mobility limitation in the elderly. J. Am. Geriatr. Soc. 2004, 52, 1105-1113. [CrossRef] [PubMed]

19. Rendina, D.N.; Ryff, C.D.; Coe, C.L. Precipitous dehydroepiandrosterone declines reflect decreased physical vitality and function. J. Gerontol. Ser. A Biomed. Sci. Med. Sci. 2016, 72, 747-753. [CrossRef] [PubMed]

20. Sabour, H.; Latifi, S.; Soltani, Z.; Shakeri, H.; Norouzi Javidan, A.; Ghodsi, S.; Hadian, M.R.; Emami Razavi, S. C-reactive protein as an available biomarker determining mental component of health-related quality of life among individuals with spinal cord injury. J. Spinal Cord Med. 2017, 40, 329-337. [CrossRef]

21. Smagula, S.F.; Ancoli-Israel, S.; Barrett-Connor, E.; Lane, N.E.; Redline, S.; Stone, K.L.; Cauley, J.A. Osteoporotic Fractures in Men (MrOS) Research Group Inflammation, sleep disturbances, and depressed mood among community-dwelling older men. J. Psychosom. Res. 2014, 76, 368-373. [CrossRef] [PubMed]

22. Won, C.W.; Lee, S.; Kim, J.; Chon, D.; Kim, S.; Kim, C.; Kim, M.K.; Cho, B.; Choi, K.M.; Roh, E. Korean frailty and aging cohort study (KFACS): Cohort profile. BMJ Open 2020, 10, e035573. [CrossRef]

23. Kim, M.K.; Lee, W.; Kang, J.; Kang, J.; Kim, B.T.; Kim, S.M.; Kim, E.M.; Suh, S.; Shin, H.J.; Lee, K.R. 2014 clinical practice guidelines for overweight and obesity in Korea. Endocrinol. Metab. 2014, 29, 405-409. [CrossRef] [PubMed]

24. Lee, Y.H.; Lee, K.J.; Han, G.S.; Yoon, S.J.; Lee, Y.K.; Kim, C.H.; Kim, J.L. The Development of Physical Functioning Scale for Community-Dwelling Older Persons. J. Prev. Med. Public Health 2002, 35, 359-374.

25. Yoon, H.S.; Yang, J.J.; Song, M.; Lee, H.W.; Han, S.; Lee, S.A.; Choi, J.Y.; Lee, J.K.; Kang, D. Correlates of self-reported sleep duration in middle-aged and elderly Koreans: From the Health Examinees Study. PLoS ONE 2015, 10, e0123510. [CrossRef]

26. Busija, L.; Pausenberger, E.; Haines, T.P.; Haymes, S.; Buchbinder, R.; Osborne, R.H. Adult measures of general health and health-related quality of life: Medical Outcomes Study Short Form 36-Item (SF-36) and Short Form 12-Item (SF-12) Health Surveys, Nottingham Health Profile (NHP), Sickness Impact Profile (SIP), Medical Outcomes Study Short Form 6D (SF-6D), Health Utilities Index Mark 3 (HUI3), Quality of Well-Being Scale (QWB), and Assessment of Quality of Life (AQOL). Arthritis Care Res. 2011, 63, S383-S412.

27. Bacci, M.R.; Adami, F.; Figueiredo, F.; Alves, B.; da Veiga, G.L.; Fonseca, F. Quality of life on hemodialysis and inflammation: A descriptive analysis. Braz. J. Med. Biol. Res. 2018, 51. [CrossRef]

28. Kim, S.; Jo, M.; Ahn, J.; Ock, M.; Shin, S.; Park, J. Assessment of psychometric properties of the Korean SF-12 v2 in the general population. BMC Public Health 2014, 14, 1086. [CrossRef]

29. Almutary, H.; Douglas, C.; Bonner, A. Towards a symptom cluster model in chronic kidney disease: A structural equation approach. J. Adv. Nurs. 2017, 73, 2450-2461. [CrossRef]

30. Gift, A.G.; Jablonski, A.; Stommel, M.; Given, C.W. Symptom Clusters in Elderly Patients with Lung Cancer. Oncol. Nurs. Forum. 2004, 31, 202-212.

31. Conley, S.; Jeon, S.; Proctor, D.D.; Sandler, R.S.; Redeker, N.S. Longitudinal changes in symptom cluster membership in inflammatory bowel disease. J. Nurs. Scholarsh. 2018, 50, 473-481. [CrossRef] [PubMed]

32. Almutary, H.; Bonner, A.; Douglas, C. Which patients with chronic kidney disease have the greatest symptom burden? A comparative study of advanced CKD stage and dialysis modality. J. Ren. Care 2016, 42, 73-82. [CrossRef] [PubMed] 
33. Li, H.; Xie, L.; Yang, J.; Pang, X. Symptom burden amongst patients suffering from end-stage renal disease and receiving dialysis: A literature review. Int. J. Nurs. Sci. 2018, 5, 427-431. [CrossRef]

34. Lehto, R.H. Symptom burden in lung cancer: Management updates. Lung Cancer Manag. 2016, 5, 61-78. [CrossRef]

35. Steinberg, B.A.; Piccini JP, S. Tackling Patient-Reported Outcomes in Atrial Fibrillation and Heart Failure: Identifying DiseaseSpecific Symptoms? Cardiol. Clin. 2019, 37, 139-146. [CrossRef] [PubMed]

36. Eckerblad, J.; Theander, K.; Ekdahl, A.W.; Jaarsma, T. Symptom trajectory and symptom burden in older people with multimorbidity, secondary outcome from the RCT AG e-FIT study. J. Adv. Nurs. 2016, 72, 2773-2783. [CrossRef] [PubMed]

37. Patel, K.V.; Guralnik, J.M.; Phelan, E.A.; Gell, N.M.; Wallace, R.B.; Sullivan, M.D.; Turk, D.C. Symptom burden among communitydwelling older adults in the United States. J. Am. Geriatr. Soc. 2019, 67, 223-231. [CrossRef] [PubMed]

38. Layne, A.S.; Hsu, F.; Blair, S.N.; Chen, S.; Dungan, J.; Fielding, R.A.; Glynn, N.W.; Hajduk, A.M.; King, A.C.; Manini, T.M. Predictors of change in physical function in older adults in response to long-term, structured physical activity: The LIFE Study. Arch. Phys. Med. Rehabil. 2017, 98, 11-24.e3. [CrossRef]

39. Ory, M.G.; Towne, S.D.; Won, J.; Forjuoh, S.N.; Lee, C. Social and environmental predictors of walking among older adults. BMC Geriatr. 2016, 16, 155. [CrossRef] [PubMed]

40. Cimas, M.; Ayala, A.; Sanz, B.; Agulló-Tomás, M.S.; Escobar, A.; Forjaz, M. Chronic musculoskeletal pain in European older adults: Cross-national and gender differences. Eur. J. Pain 2018, 22, 333-345. [CrossRef]

41. Cheng, H.; Chen, C.; Lin, M.; Wang, C.; Yang, Y.; Lu, F.; Wu, J.; Lin, S. Gender differences in the relationship between walking activity and sleep disturbance among community-dwelling older adult with diabetes in Taiwan. J. Women Aging 2019, 31, 108-116. [CrossRef]

42. Choi, K.; Jeon, G.; Jang, K. Gender differences in the impact of intergenerational support on depressive symptoms among older adults in korea. Int. J. Environ. Res. Public Health 2020, 17, 4380. [CrossRef]

43. Tsunoda, K.; Soma, Y.; Kitano, N.; Tsuji, T.; Mitsuishi, Y.; Yoon, J.; Okura, T. Age and gender differences in correlations of leisure-time, household, and work-related physical activity with physical performance in older J apanese adults. Geriatr. Gerontol. Int. 2013, 13, 919-927. [CrossRef]

44. Yong, V.; Saito, Y.; Chan, A. Gender differences in health and health expectancies of older adults in Singapore: An examination of diseases, impairments, and functional disabilities. J. Cross-Gult. Gerontol. 2011, 26, 189-203. [CrossRef]

45. Son, Y.; Lee, K.; Kim, B. Gender differences in the association between frailty, cognitive impairment, and self-care behaviors among older adults with atrial fibrillation. Int. J. Environ. Res. Public Health 2019, 16, 2387. [CrossRef] [PubMed]

46. Song, H.; Park, H.; Yun, K.; Cho, S.; Choi, E.; Lee, S.; Kim, J.; Sung, H.; Choi, S.; Yoon, Y. Gender and age differences in the impact of overweight on obesity-related quality of life among Korean adults. Obes. Res. Clin. Pract. 2010, 4, e15-e23. [CrossRef] [PubMed]

47. Yun, M.; Kim, E. Gender difference in the association between alcohol consumption and depressive symptoms among the elderly in rural areas. J. Ethn. Subst. Abus. 2019, 1-19. [CrossRef] [PubMed]

48. Storer, T.W.; Basaria, S.; Traustadottir, T.; Harman, S.M.; Pencina, K.; Li, Z.; Travison, T.G.; Miciek, R.; Tsitouras, P.; Hally, K. Effects of testosterone supplementation for 3 years on muscle performance and physical function in older men. J. Clin. Endocrinol. Metab. 2017, 102, 583-593. [CrossRef] [PubMed]

49. Walther, A.; Tsao, C.; Pande, R.; Kirschbaum, C.; Field, E.; Berkman, L. Do dehydroepiandrosterone, progesterone, and testosterone influence women's depression and anxiety levels? Evidence from hair-based hormonal measures of 2105 rural Indian women. Psychoneuroendocrinology 2019, 109, 104382. [CrossRef] [PubMed]

50. Zitzmann, M. Testosterone, mood, behaviour and quality of life. Andrology 2020, 8, 1598-1605. [CrossRef] [PubMed]

51. Nam, Y.; Lee, G.; Yun, J.M.; Cho, B. Testosterone replacement, muscle strength, and physical function. World J. Men's Health 2018, 36, 110-122. [CrossRef]

52. Davis, S.R.; Panjari, M.; Stanczyk, F.Z. Clinical review: DHEA replacement for postmenopausal women. J. Clin. Endocrinol. Metab. 2011, 96, 1642-1653. [CrossRef]

53. Peixoto, C.; Grande, A.J.; Mallmann, M.B.; Nardi, A.E.; Cardoso, A.; Veras, A.B. Dehydroepiandrosterone (DHEA) for depression: A systematic review and meta-analysis. Cns Neurol. Disord. Drug Targets (Former. Curr. Drug Targets-Cns Neurol. Disord.) 2018, 17, 706-711. [CrossRef]

54. Li, Y.; Ren, J.; Li, N.; Liu, J.; Tan, S.C.; Low, T.Y.; Ma, Z. A dose-response and meta-analysis of dehydroepiandrosterone (DHEA) supplementation on testosterone levels: Perinatal prediction of randomized clinical trials. Exp. Gerontol. 2020, $141,111110$. [CrossRef] [PubMed]

55. Xie, M.; Zhong, Y.; Xue, Q.; Wu, M.; Deng, X.; Santos, H.O.; Tan, S.C.; Kord-Varkaneh, H.; Jiao, P. Impact of dehydroepianrosterone (DHEA) supplementation on serum levels of insulin-like growth factor 1 (IGF-1): A dose-response meta-analysis of randomized controlled trials. Exp. Gerontol. 2020, 136, 110949. [CrossRef] [PubMed] 